\title{
Avaliação por competência em cursos online abertos e massivos por meio de rubrica
}

\section{Competency based evaluation in massive open online courses by the use of rubric}

\author{
Byanca Neumann Salerno, ${ }^{1}$, Maria do Carmo Duarte Freitas ${ }^{1}$ \\ ${ }^{1}$ Universidade Federal do Paraná, Curitiba, PR, Brasil
}

Autor para correspondência/Mail to: Byanca Neumann Salerno, byancaa.s2@gmail.com

Financiamento/Funding: Fundação Araucária e Governo do Estado do Paraná

Copyright (C) 2019 Salerno \& Freitas. Todo o conteúdo da Revista (incluindo-se instruções, política editorial e modelos) está sob uma licença Creative Commons Atribuição-NãoComercial-Compartilhalgual 3.0 Não Adaptada. Ao serem publicados por esta Revista, os artigos são de livre uso em ambientes educacionais, de pesquisa e não comerciais, com atribuição de autoria obrigatória. Mais informações em http://revistas.ufpr.br/atoz/about/submissions\#copyrightNotice.

\begin{abstract}
Resumo
O desenvolvimento das tecnologias da informação e seu uso na área da educação contextualiza o surgimento dos Cursos Online Abertos e Massivos caracterizados pela ausência de pré-requisitos para ingresso e quantidade ilimitada de estudantes. Um dos problemas enfrentados nesses cursos é a alta taxa de evasão dos estudantes devido à falta de engajamento. Além disso, há dificuldade para avaliar a aprendizagem e garantir que as competências esperadas com o curso sejam desenvolvidas. Esta pesquisa tem como objetivo formular diretrizes para o desenvolvimento de conteúdos em MOOCs com a utilização de rubricas de avaliação por competências. A metodologia é de revisão sistemática a partir de pesquisa bibliográfica e análise de conteúdo sobre as práticas e estratégias educacionais utilizadas no ensino superior e nos Cursos Online Abertos e Massivos. Os resultados parciais mostram que a avaliação é capaz de motivar o engajamento dos estudantes e que grandes provedores de MOOCs como o Coursera e o edX têm investido em tecnologias automáticas para esse fim. No entanto, essas tecnologias não avaliam os estudantes a partir de competências. Portanto, elas não são suficientes para substituir a avaliação formativa baseada em competências que o professor é capaz de proporcionar por meio de rubricas.
\end{abstract}

Palavras-chave: MOOCs; Avaliação por competências; Rubricas

\begin{abstract}
The development of information technology and its use in education contextualizes the emergence of Massive Open Online Courses characterized by the absence of prerequisites for admission and unlimited number of students. One of the problems faced in these courses is the high dropout rate of students due to lack of engagement. In addition, there is difficulty in assessing learning and ensuring that the skills expected from the course are developed. This research aims to formulate guidelines for the development of content in MOOCs with the use of competency evaluation rubrics. The methodology is a systematic review based on bibliographic research and content analysis on the educational practices and strategies used in higher education and Massive Open Online Courses. The partial results show that the evaluation is able to motivate student engagement and that large MOOC providers such as Coursera and edX have invested in automatic technologies for this purpose. However, these technologies do not evaluate students from skills. Therefore, they are not enough to replace the competency-based training assessment that the teacher is able to provide by the use of rubrics.
\end{abstract}

Keywords: MOOCs; Competency based evaluation; Rubrics.

\section{INTRODUÇÃO}

A sociedade da informação e do conhecimento trouxe mudanças aos ambientes organizacionais. Cada vez mais buscam-se profissionais competentes para atuar em suas áreas do conhecimento, independentemente de sua natureza. Competências são um conjunto de conhecimentos, habilidades e atitudes (Fleury \& Fleury, 2001) capazes de serem aprendidas em contexto acadêmico ou organizacional e necessárias para o desempenho de funções.

A formação por competências é uma prática cada vez mais comum em países desenvolvidos como os Estados Unidos e membros da União Europeia (Siebiger, 2011). A avaliação por competências é capaz de identificar falhas no processo de ensino e propor melhorias para alcançar os objetivos institucionais. Nesse contexto, a rubrica é um modelo de avaliação por competências caracterizado pela flexibilidade de sua construção e focado em avaliar aspectos qualitativos a partir de critérios pré-estabelecidos (Velozo, 2014).

A flexibilização do ensino com o uso das Tecnologias da Informação e Comunicação (TICs) intensifica o ensino online. Desde 2008 os Cursos Abertos Online e Massivos (MOOCs) têm conquistado espaço nas universidades (Haber, 2014). Esses cursos são caracterizados pela ausência de pré-requisitos e de limite na quantidade de estudantes (Andrade \& Silveira, 2016). Essas características os tornam atrativos para o público, independentemente de localização geográfica, classe social ou escolaridade. Apesar de haver procura, um dos problemas verificados é taxa de evasão nos MOOCs. Menos de 10

Jordan (2013) aponta que a avaliação é um elemento motivador da aprendizagem e que o uso de tecnologias para avaliação online é útil principalmente em salas de aula com grande número de estudantes. Há dificuldade para avaliar nos MOOCs, tendo em vista a quantidade de estudantes que eles suportam. Geralmente as avaliações empregadas são auto avaliação e avaliação pelos pares, entretanto esses dois tipos restringem-se à medição dos 
conhecimentos adquiridos de maneira quantitativa (Staubitz, Petrick, Bauer, Renz \& Meinel, 2016). Para além dos conhecimentos adquiridos, o professor, como mediador do aprendizado, deve ser capaz de incentivar e criar condições para proporcionar o engajamento do estudante e garantir o desenvolvimento de competências alinhadas às demandas do mercado de trabalho.

Nos MOOCs a necessidade de estimular os estudantes torna-se ainda maior, em razão da condição virtual de aprendizagem que possui características diversas da presencial. O ensino online exige do aluno, mas também exige do professor, que precisa ter competência no uso das TICs, assim como para inovar nas práticas pedagógicas e adequá-las para as necessidades de seus estudantes. Desse modo, a formação continuada de professores é uma condição para que haja mudança em práticas pedagógicas (Perin, 2017).

A literatura sobre MOOCs no contexto do ensino superior ganhou importância a partir de 2012 (Andrade \& Silveira, 2016). Problemas como evasão e falta de engajamento e participação dos estudantes são associados a esses cursos com frequência (Andrade \& Silveira, 2016; Carvalho, Junqueira, Pinheiro, Fernandes, \& Cruz, 2013; Silva, 2017). A avaliação online é capaz de motivar e engajar estudantes na medida em que provê feedback sobre a aprendizagem e permite que os estudantes melhorem seu desempenho (Jordan, 2013). A própria construção dos MOOCs deve estimular o engajamento e aprendizagem e sustentar avaliação e mensuração dos resultados da aprendizagem (Chauhan, 2014), visto que menos de 10

No cenário internacional, pesquisas apontam que a avaliação em MOOCs geralmente se restringe à auto avaliação do estudante e à avaliação pelos pares, sendo que junto a esses dois tipos podem se utilizar rubricas (Staubitz et al., 2016). Apesar da utilização desses métodos, seu foco não é formativo, ou seja, nas dificuldades ao longo do estudo e nas competências desenvolvidas pelo estudante. Em virtude da escalabilidade dos MOOCs que confere a eles quantidades ilimitadas de estudantes, existe um desafio para que a avaliação proporcione um retorno construtivo para a aprendizagem.

A avaliação pelos pares tem sido utilizada como forma de se resolver esse problema, mas nem sempre os pares estão capacitados para avaliar (Staubitz et al., 2016). Geralmente o professor se limita a avaliações de múltipla-escolha, questões de certo ou errado e de vocabulário, que são facilmente avaliadas mediante computador de maneira automática. As avaliações escritas, por sua vez, têm se mostrado um desafio, já que a avaliação automática não tem a mesma interpretação do professor (Balfour, 2013; Hew \& Cheung, 2014).

Dessa forma, a avaliação em MOOCs se limita aos conhecimentos adquiridos pelo estudante e não necessariamente às competências desenvolvidas durante a aprendizagem. Os estudos sobre a avaliação da aprendizagem em MOOCs no contexto do ensino superior ainda são pouco explorados (Andrade \& Silveira, 2016). Além disso, a avaliação por competências ainda não é uma prática consolidada no Brasil, onde predominam as avaliações quantitativas de desempenho (Bianchi, 2013).

O Censo EAD 2016 mostrou que a principal preocupação apontada pelas instituições de ensino que ofertam essa modalidade é a inovação em abordagens pedagógicas (Associação Brasileira de Educação a Distância, 2017). Além disso, O Ministério da Educação (MEC) em seus referenciais de qualidade para o EaD orienta que a avaliação seja predominantemente qualitativa voltada para o desenvolvimento de competências (Ministério da Educação, 2007).

Dessa forma, esta pesquisa justifica-se pela necessidade diminuir os altos índices de evasão nos MOOCs e ao mesmo tempo fornecer uma aprendizagem significativa baseada em competências. Para isso, é necessário identificar práticas e estratégias educacionais utilizadas no ensino superior e em MOOCs de forma que seja possível formular diretrizes para que os professores estejam capacitados para desenvolver conteúdo baseado em competências e avaliá-los por meio de rubrica.

A avaliação por competências mediada por rubrica gera dados e informações que subsidiam a tomada de decisão dos professores e coordenadores da educação quanto à capacitação de seus professores e mudança de práticas pedagógicas que atendam às necessidades atuais da educação brasileira. Os MOOCs são considerados uma tecnologia emergente no ensino superior e no Brasil ainda não há estudos aprofundados sobre o tema. As pesquisas em relação à docência evidenciam que existe uma necessidade de formação continuada dos professores para a construção de conteúdos e de estratégias de interação com os estudantes (Andrade \& Silveira, 2016).

Tendo em vista os problemas mencionados, pretende-se responder ao seguinte questionamento: De que forma desenvolver conteúdos em MOOCs a partir de rubricas para estimular o engajamento dos discentes? Para responder a este problema o objetivo geral desta pesquisa é: formular diretrizes para o desenvolvimento de conteúdos em MOOCs com a utilização de rubricas de avaliação. E para atingir esse objetivo buscar-se-á na literatura boas práticas educacionais utilizadas em MOOCs; tecnologias e estratégias didáticas no ensino universitário; e a forma de construção de rubricas para conteúdo teórico e prático. 


\section{REVISÃO DE LITERATURA}

Massive Open Online Courses (MOOCs) ou Cursos Online Abertos e Massivos são uma modalidade de curso online sem limites quanto à quantidade e localização física de estudantes, característica que confere o termo massivo; e ausência de pré-requisitos, característica que confere o termo aberto. Na prática, existem controvérsias quanto à definição dos MOOCs, uma vez que existem cursos com essa denominação que possuem apenas uma das características anteriores (Andrade \& Silveira, 2016).

Em teoria, qualquer pessoa pode iniciar o curso sem pré-requisitos e sem custos, embora exista a possibilidade de adquirir um certificado válido de participação mediante o pagamento de um determinado valor. Além disso, é necessário um mínimo de conhecimento do uso de tecnologia e acesso à internet por meio de dispositivo móvel ou computador (Andrade \& Silveira, 2016). Os MOOCs têm sido aplicados como "complemento às aulas tradicionais, nivelamento acadêmico, educação corporativa e processos de formação continuada e de desenvolvimento institucionais" (Andrade \& Silveira, 2016, p. 106), sendo a formação continuada comum entre professores.

O primeiro curso a ser denominado como MOOC foi o Connectivism and Connective Knowledge ministrado por Stephen Downes e George Siemens da Universidade do Athabasca, no Canadá, em 2008. A popularidade dessa modalidade de ensino, entretanto, começou em 2012 quando o New York Times publicou que esse foi o ano dos MOOCs.

Entre 2011 e 2012 surgiram as três maiores empresas provedoras de MOOCs do mundo. A KnowLabs, hoje conhecida como Udacity, foi fundada por Sebastian Thrun, professor de ciência da computação da Universidade de Stanford nos Estados Unidos. Seu primeiro curso sobre inteligência artificial alcançou 160 mil estudantes. Andrew Ng e Daphne Koller da mesma universidade alcançaram 100 mil participantes em seu MOOC sobre machine learning. Esses dois professores deram origem à plataforma Coursera (Haber, 2014).

Embora essas informações conduzam ao pensamento de que esse fenômeno da educação aberta surgiu com os MOOCs, cerca de uma década antes o Massachusetts Institute of Technology (MIT) já disponibilizava acesso aos seus materiais em vídeo e escritos utilizados presencialmente por meio do portal Open Course Ware. Em vez de unir-se à concorrência, o MIT, em parceria com a Universidade de Harvard, deu origem à plataforma edX. Ao final de 2013 essas três empresas já ofereciam cerca de 500 cursos para sete milhões de usuários distribuídos entre as três (Haber, 2014).

Existem na literatura duas classificações básicas para os MOOCs: cMOOC e xMOOC. O primeiro é fundamentado no conectivismo e a aprendizagem é construída colaborativamente enquanto o segundo é focado na transmissão de conteúdo (Andrade \& Silveira, 2016). É comum nos cMOOCs que o estudante interaja com os outros por meio das redes sociais e tenha autonomia para escolher o que deseja aprender, sendo que nenhum conteúdo tem natureza obrigatória e não há objetivos a serem alcançados (Carvalho et al., 2013). Já o xMOOC funciona semelhante ao ensino presencial em que o conteúdo é transmitido e são feitas avaliações. Além disso, em comparação com o tipo conectivista, a interação entre os usuários no tipo "tradicional" é incipiente (Andrade \& Silveira, 2016).

Ambos os modelos possuem falhas e acertos. Um dos problemas apresentados por Carvalho et al. (2013, p. 209) em relação aos cMOOCs é que a ausência de tradicionais objetivos de aprendizagem e de interação ordenada por um professor podem gerar uma sensação de falta de orientação aos participantes, habituados às atividades desenvolvidas em contexto de ensino.

Pelo mesmo motivo, as trocas simultâneas realizadas nestes cursos podem gerar uma sobrecarga cognitiva para o aluno e o alto nível de autonomia da aprendizagem exigido deles pode impulsionar a evasão. Andrade e Silveira (2016) complementam que os cMOOCs precisam de elementos que motivem os estudantes a partir da participação, interação e colaboração.

As primeiras iniciativas de MOOC no Brasil foram em 2012 por meio da Unesp Aberta, que oferece cursos em diversos temas e do Portal Veduca, que lançou o primeiro curso de especialização totalmente aberto e online do mundo (Andrade \& Silveira, 2016). Em relação ao número de inscritos, o país está em terceiro lugar, atrás dos Estados Unidos e da Índia (Silva, 2017). Esses dados mostram que o público brasileiro tem bastante aderência a esse modelo. Além dessas iniciativas, no Brasil os MOOCs têm sido utilizados também na formação de professores e para o oferecimento de disciplinas híbridas associadas ao ensino presencial, que servem como complemento ao que é estudado em sala de aula (Silva, 2017).

\section{PROCEDIMENTOS METODOLÓGICOS}

Esta pesquisa caracteriza-se quanto ao propósito como exploratória, uma vez que as pesquisas sobre MOOCs ainda são incipientes no contexto do ensino superior e não há foco no desenvolvimento de competências. A natureza dos dados é qualitativa, pois baseia sua investigação na própria literatura. 
Quanto à forma é uma pesquisa não experimental e bibliográfica. Os procedimentos de coleta dar-se-ão por meio de revisão sistemática da literatura sobre MOOCs e ensino superior para identificar estratégias e práticas educacionais no período de 2008 a 2018 nos idiomas inglês, português e espanhol nas bases de dados disponíveis no Portal de Periódicos da Capes, Ebsco e Elsevier. Pretende-se investigar também práticas para a construção de rubricas de avaliação para conteúdo teórico e prático. O tratamento dos dados será feito mediante a técnica de análise de conteúdo. O cronograma do desenvolvimento da pesquisa é apresentado adiante (Quadro 1).

\begin{tabular}{|c|c|c|c|c|c|c|c|c|c|c|}
\hline \multicolumn{11}{|c|}{2018} \\
\hline & mar & $a b r$ & mai & jun & jul & ago & set & out & nov & dez \\
\hline Início do mestrado & $\mathrm{x}$ & & & & & & & & & \\
\hline Introdução & & $x$ & $x$ & $x$ & $x$ & $x$ & & & & \\
\hline Problema de Pesquisa & & $x$ & $x$ & $x$ & $x$ & $x$ & & & & \\
\hline Objetivos & & $x$ & $x$ & $x$ & & & & & & \\
\hline Justificativa & & $x$ & $x$ & $x$ & $x$ & $x$ & & & & \\
\hline Referencial Teórico & & & $X$ & $x$ & $x$ & $x$ & $x$ & $x$ & $x$ & \\
\hline Metodologia & & & $\mathrm{x}$ & $\mathrm{x}$ & $x$ & $\mathrm{x}$ & & & & \\
\hline \multicolumn{11}{|c|}{2019} \\
\hline Coleta de Dados & $x$ & $x$ & $x$ & & & & & & & \\
\hline Tratamento de Dados & $x$ & $x$ & $x$ & & & & & & & \\
\hline Análise de Dados & & & $x$ & $x$ & $x$ & $x$ & & & & \\
\hline Resultados Parciais & & & $x$ & $x$ & & & & & & \\
\hline Qualificação & & & & $x$ & & & & & & \\
\hline Ajustes & & & & $x$ & $x$ & $x$ & $x$ & & & \\
\hline Resultados Totais & & & $x$ & $x$ & $x$ & $x$ & $x$ & $x$ & & \\
\hline Considerações & & & & & & & $x$ & $x$ & $x$ & \\
\hline Defesa estimada & & & & & & & & & & $x$ \\
\hline
\end{tabular}

Quadro 1. Cronograma estimado da pesquisa

Fonte: Elaborado pelas autoras (2018).

\section{CONSIDERAÇÕES FINAIS}

Constatou-se na literatura que a avaliação é uma estratégia capaz de estimular o engajamento dos estudantes, uma vez que os motiva a melhorar seu desempenho (Jordan, 2013). No contexto dos MOOCs identificaram-se dois tipos predominantes: auto avaliação e avaliação pelos pares (Staubitz et al., 2016). A avaliação pelos pares sofre críticas quanto à sua validade e confiabilidade, pois o feedback e a capacidade de julgamento dos estudantes são questionados pelo fato de nem todos estarem preparados para avaliar (Staubitz et al., 2016). Por outro lado, essa forma de avaliação aumenta a interação entre estudantes e entre esses e o professor (Rosa, Coutinho \& Flores, 2017).

Ainda que não haja consenso sobre esse método ser ou não adequado, é convergente a opinião que ele ajuda a administrar turmas com quantidade de estudantes ilimitada, que geram volume de trabalho além das capacidades do professor. O fato de um estudante avaliar seu par ou pares faz com que ele desenvolva competências como: trabalho colaborativo, responsabilidade, comunicação, liderança, gestão do tempo, resolução de problemas e tomada de decisão, além de compreender e refletir sobre os assuntos estudados.

A aprendizagem depende, em parte, da compreensão de como o processo avaliativo ocorre. Por essa razão, a forma de avaliar precisa estar clara quanto aos seus objetivos (Rosa, Coutinho \& Flores, 2017). A organização e distribuição dos pares varia conforme a quantidade de estudantes e a estratégia do professor. Rosa, Coutinho e Flores (2017) constataram diferentes experiências quanto à estratégia de avaliação. A abordagem varia entre quantitativa (por escalas), qualitativa (oral ou escrita) e mista.

Existe uma dificuldade em relação à avaliação escrita, mas alguns provedores de MOOCs como o edX já implementaram uma aplicação automática baseada em machine learning denominada Automated Essay Scoring (AES) para avaliar a aprendizagem escrita (Balfour, 2013). Esse tipo de avaliação provê feedback sobre gramática, vocabulário, trechos irrelevantes, estilo, complexidade, conclusões, entre outros. Embora tenha mostrado resultados satisfatórios (Balfour, 2013), percebe-se uma abordagem de avaliação diagnóstica, cujo foco não é o desenvolvimento de competências, pois as máquinas não possuem nível de compreensão e abstração semelhante ao de seres humanos.

Outra aplicação utilizada em MOOCs é o Calibrated Peer Review (CPR) utilizado pelo Coursera, na qual é feita auto avaliação e avaliação pelos pares e elas são comparadas com uma rubrica que destaca os pontos desejados pelo professor. O CPR tem alcançado resultados de melhoria na aprendizagem dos estudantes e desenvolvimento de competências de escrita e habilidade de avaliação. Entretanto, essa ferramenta não suporta 
grandes quantidades de estudantes ou textos muito longos (Balfour, 2013). Portanto, percebe-se que embora os esforços tenham se voltado para tecnologias automáticas, elas não são suficientes para substituir a avaliação formativa baseada em competências que o professor é capaz de proporcionar.

\section{AGRADECIMENTOS}

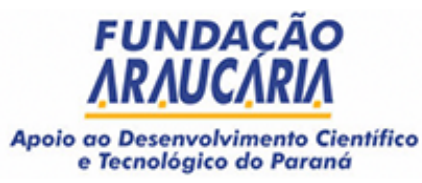

FUNDACÃO

poio ao Desenvolvimento Cientifico

e Tecnológico do Parane

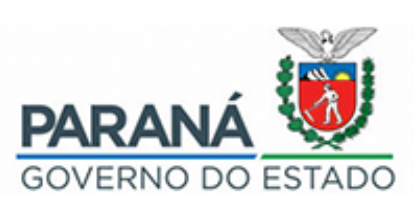

À Fundação Araucária e ao Governo do Estado pelo apoio financeiro para a realização do evento.

\section{REFERÊNCIAS}

Andrade, M. V. M., \& Silveira, I. F. (2016). Panorama da aplicação de massive open online course (MOOC) no ensino superior: desafios e possibilidades. EaD em foco, 6(3), 101-114. Recuperado de https://goo.gl/pbGxny

Associação Brasileira de Educação a Distância. (2017). Censo EAD Brasil 2016: relatório analítico da aprendizagem a distância no Brasil. Recuperado de https://goo.gl/dHTTYY

Balfour, S. P. (2013). Assessing writing in MOOCs: automated essay scoring and calibrated peer review. Research 8 Practice in Assessment, 8, 40-48. Recuperado de https://goo.gl/kAR4hm

Bianchi, P. C. F. (2013). Avaliação da aprendizagem na educação a distância. Dissertação de Mestrado, Universidade Federal de São Carlos, Brasil. Recuperado de https://goo.gl/AN5Dgm

Carvalho, R. L., Junqueira, E. S., Pinheiro, J. L., Fernandes, J. H. M., \& Cruz, M. C. F. (2013). A cibercultura e os MOOCs: análise da interação dos alunos em duas experiências no Brasil. Revista EDaPECI, 13(2), 200-215. Recuperado de https://seer.ufs.br/index.php/edapeci/article/view/1688

Chauhan, A. (2014). Massive open online courses (MOOCS): emerging trends in assessment and accreditation. Digital Education Review, 25, 7-18. Recuperado de http://greav.ub.edu/der/http://greav.ub.edu/der/

Fleury, M. T. L., \& Fleury, A. (2001). Construindo o conceito de competência. Revista de Administração Contemporânea, 5(spe), 183-196. doi: 10.1590/s1415-65552001000500010

Haber, J. (2014). MOOCs. London: The MIT Press.

Hew, K. F., \& Cheung, W. S. Students' and instructors' use of massive open online courses (MOOCs): motivations and challenges. Educational Research Review, 12, 45-58. Recuperado de: https://goo.gl/fTGsGY

Jordan, S. (2013). E-assessment: past, present and future. Raine, NDIR, 9(1), 87-106. Recuperado de https://goo.gl/bkFiK7

Ministério da Educação. (2007). Referenciais de qualidade para EaD. Brasília. Recuperado de http://portal.mec.gov.br/seed/arquivos/pdf/legislacao/refead1.pdf

Perin, E. S. (2017). Competências docentes digitais para o compartilhamento de práticas e recursos educacionais. Dissertação de Mestrado, Universidade Federal do Paraná, Brasil. Recuperado de https://goo.gl/Nebw2S

Rosa, S. S., Coutinho, C. P., / Flores, M. A. (2017). Online peer assessment no ensino superior: uma revisão sistemática da literatura em práticas educacionais. Avaliação: Revista da Avaliação da Educação Superior, 22(1), 55-83. Recuperado de https://goo.gl/gMdrty

Siebiger, R. H. (2011). Influências do processo de Bolonha europeu nas políticas de educação superior brasileiras e na criação de Universidades federais. XXV Simpósio Brasileiro. Recuperado de https://goo.gl/aSJZmn

Silva, H. S. (2017). Revisão sistemática sobre o uso dos MOOCS no Brasil. Artigo de especialização, Universidade Federal de Santa Maria, Brasil. Recuperado de http://repositorio.ufsm.br/handle/1/12046

Staubitz, T., Petrick, D., Bauer, M., Renz, J., \& Meinel, C. (2016). Improving the Peer Assessment Experience on MOOC Platforms. Proceedings Of The Third ACM Conference On Learning. doi: 10.1145/2876034.2876043

Velozo, A. V. (2014). Avaliação por competência no domínio de língua estrangeira: uma aplicação profissional mediada por rubrica. Dissertação de mestrado, Universidade Federal do Paraná, Brasil. Recuperado de https://goo.gl/AqtW5y 